\title{
Outcomes in Patients with Staphylococcus aureus Bacteremia Treated with Dalbavancin in Clinical Trials
}

\author{
Pedro L. Gonzalez • Urania Rappo · Karthik Akinapelli • \\ Jennifer S. McGregor · Sailaja Puttagunta $\cdot$ Michael W. Dunne
}

Received: April 16, 2021 / Accepted: November 12, 2021 / Published online: December 14, 2021

(C) The Author(s) 2021

\begin{abstract}
Introduction: Dalbavancin is a long-acting, bactericidal, lipoglycopeptide antibiotic approved by the US Food and Drug Administration and the European Medicines Agency for treatment of acute bacterial skin and skin structure infections in adults, with potent activity against Gram-positive pathogens,
\end{abstract}

Pedro L. Gonzalez and Urania Rappo contributed equally to the work.

P. L. Gonzalez $(\varangle) \cdot$ S. Puttagunta $\cdot$ M. W. Dunne Infectious Disease Medical Affairs and Internal Medicine, AbbVie, Madison, NJ, USA

e-mail: papitin@yahoo.com

U. Rappo

Clinical Development, Infectious Diseases, AbbVie, Madison, NJ, USA

K. Akinapelli · J. S. McGregor

Medical Affairs Infectious Diseases, AbbVie,

Madison, NJ, USA

Present Address:

P. L. Gonzalez

Becton-Dickinson, 1 Becton Dr, Franklin Lakes,

NJ 07417-1880, USA

Present Address:

U. Rappo · S. Puttagunta

BiomX Inc., Branford, CT, USA

Present Address:

K. Akinapelli · M. W. Dunne

Iterum Therapeutics, Old Saybrook, CT, USA including methicillin-susceptible and methicillin-resistant Staphylococcus aureus. Here we describe the clearance and clinical outcomes of patients with $S$. aureus bacteremia in five clinical trials of skin and skin structure infections or catheter-related bloodstream infections that evaluated the efficacy and safety of dalbavancin.

Methods: Patients with uncomplicated S. aureus bacteremia identified in blood cultures drawn at baseline (before study drug) with at least one follow-up blood culture are described from four phase 3 trials in skin and skin structure infections and one phase 2 catheter-related infection study. Dalbavancin was administered as a single-dose (1500 mg intravenous [IV]) or a twodose regimen (1000 $\mathrm{mg}$ IV on day 1, $500 \mathrm{mg}$ IV on day 8). Comparators included vancomycin IV or linezolid IV/oral for 10-14 days.

Results: All 39 patients (100\%) who received dalbavancin, including 8 patients on the singledose regimen, had clearance of bacteremia versus 19/20 patients (95\%) treated with comparators (vancomycin or linezolid). At end of treatment, 33/36 dalbavancin-treated patients (92\%) achieved clinical success versus 18/23 patients (78\%) treated with comparators.

Conclusions: All 39 patients with uncomplicated S. aureus bacteremia treated with dalbavancin (single- or two-dose regimen) and with follow-up blood cultures had clearance of their bloodstream infection. Clinical response rates 
were similar to daily comparator therapy for 10-14 days.

Trial Registration: DISCOVER 1, NCT0133 9091; DISCOVER 2, NCT01431339; DUR001303, NCT02127970; VER001-9; VER001-4, NCT00057369.

Keywords: Acute bacterial skin and skin structures infections; Bacteremia; Dalbavancin; Gram-positive

\section{Key Summary Points}

\section{Why carry out this study?}

Dalbavancin is a long-acting, bactericidal, lipoglycopeptide antibiotic approved by the US Food and Drug Administration and the European Medicines Agency for treatment of acute bacterial skin and skin structure infections (ABSSSI) in adults.

We wished to explore the clinical outcomes and microbiological clearance in patients with ABSSSI, complicated skin and skin structure infections, or catheterrelated bloodstream infection that had Staphylococcus aureus bacteremia at baseline in phase 2 and 3 efficacy studies.

\section{What was learned from this study?}

Dalbavancin, administered as a singledose or two-dose regimen, can effectively treat uncomplicated S. aureus bacteremia in patients with ABSSSI.

\section{INTRODUCTION}

Dalbavancin is a second-generation, semisynthetic, lipoglycopeptide antibiotic structurally related to teicoplanin [1]. Dalbavancin binds to the terminal D-alanyl-D-alanine of the stem peptide in newly growing cell wall peptidoglycan, preventing cross-linking (transpeptidation and transglycosylation) of disaccharide subunits, thereby interrupting cell wall synthesis and resulting in bacterial cell death [1-3].

Dalbavancin is a long-acting, bactericidal, lipoglycopeptide antibiotic approved by the US Food and Drug Administration and the European Medicines Agency for treatment of acute bacterial skin and skin structure infections (ABSSSI) in adults [2, 3]. Dalbavancin has shown potent activity against Gram-positive pathogens responsible for ABSSSI, in particular Staphylococcus aureus, including methicillinresistant S. aureus (MRSA), streptococci, and vancomycin-susceptible Enterococcus faecalis $[4,5]$. In addition, an in vitro susceptibility study showed that dalbavancin was 4 - to 8 -fold more potent than daptomycin, and 16-fold more potent than vancomycin or linezolid against MRSA in isolates of patients with skin and skin structure infections [6]. Plasma concentrations of dalbavancin exceed bactericidal concentrations for 14 days after administration of $1500 \mathrm{mg}$ as a single dose or $1000 \mathrm{mg}$ followed by $500 \mathrm{mg}$ a week later (two-dose regimen; Fig. 1) $[3,7]$. The safety of dalbavancin has been evaluated previously in a pooled analysis of seven phase 2 and phase 3 clinical trials and was found to have overall adverse event rates similar to or lower than those for patients receiving comparator agents [8].

Phase 2 and 3 efficacy studies have been conducted and completed with dalbavancin to explore the clinical outcomes and microbiological clearance in patients with ABSSSI, complicated skin and skin structure infections (cSSSI), or catheter-related bloodstream infections $[4,5,9,10]$. This analysis focused on the patients with $S$. aureus bacteremia at baseline in these studies.

\section{METHODS}

All studies were conducted in accordance with the Declaration of Helsinki, the study protocol, the International Conference on Harmonisation tripartite guideline for Good Clinical Practice (ICH E6[R1]), and the institutional review board or ethics committee at each study site. All patients provided written informed consent before participation. 


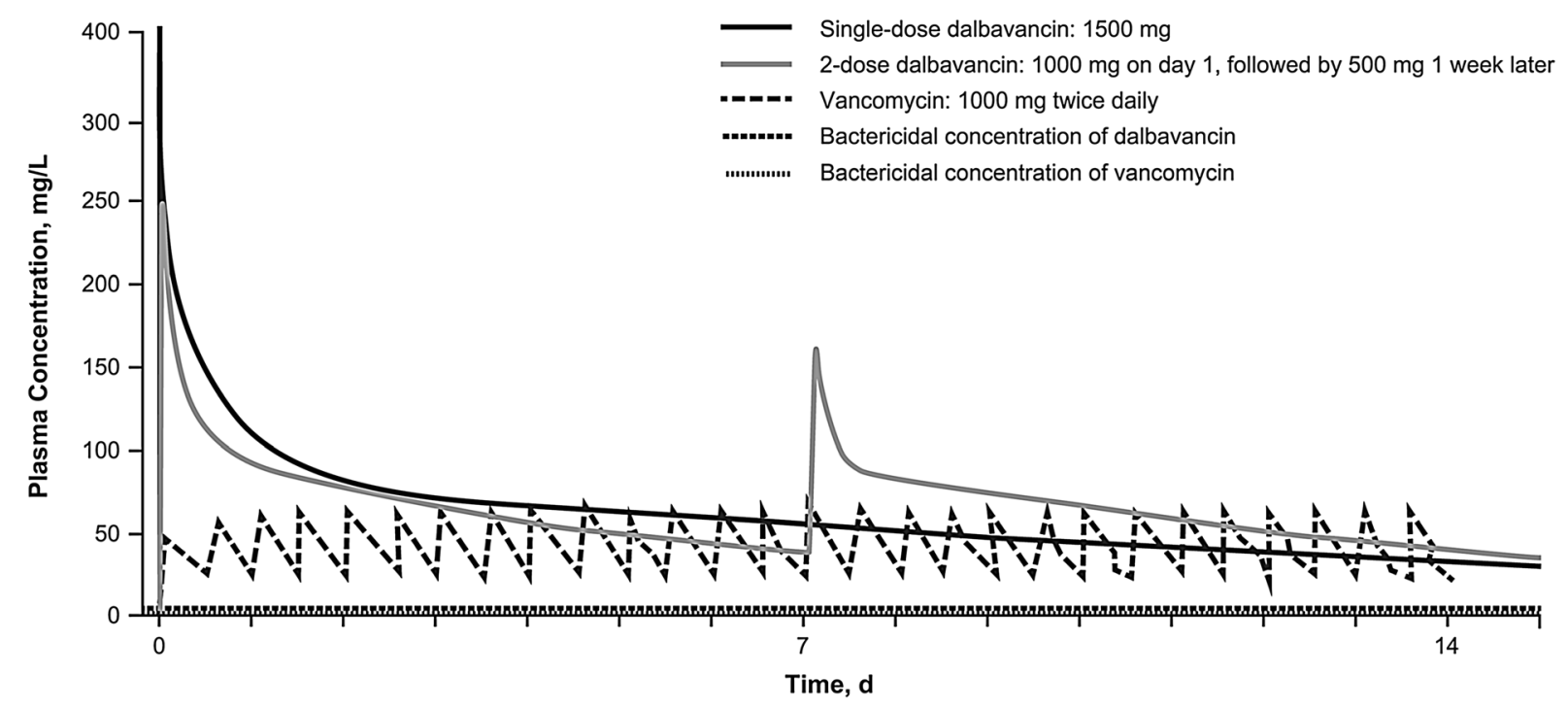

Fig. 1 Serum concentration of dalbavancin $1500 \mathrm{mg}$ after intravenous administration as a 30-min infusion in a single-dose or two-dose regimen. Adapted with permission from Dunne M and Puttagunta S. Clearance of Staphylococcus aureus bacteremia in patients treated with dalbavancin. Presented at IDWeek, October 2-6, 2013; San Francisco, CA. http://www. idweek.org

Data were analyzed from patients enrolled in the dalbavancin ABSSSI, cSSSI, or catheter-related bloodstream infection studies (Table 1) $[4,5,9,10]$. Blood cultures were drawn at baseline before study drug treatment from two different anatomical sites (not through an existing intravascular line) and then repeated every $48-72 \mathrm{~h}$ until cultures were negative. If clinically indicated, blood cultures were collected at the time of treatment discontinuation or for determination of treatment failure. Patients in whom S.aureus was identified (through the baseline blood cultures) were selected for further assessment. The catheterrelated bloodstream infection study required removal of the catheter in all patients with $S$. aureus bacteremia [9]. Data regarding clearance of bacteremia were provided for patients with at least one follow-up post-baseline blood culture. Documented clearance of bacteremia was defined by laboratory data showing followup blood culture(s) negative for the baseline blood pathogen on or before day 12 , no subsequent blood culture(s) (after the negative follow-up blood culture; if obtained) positive for the baseline blood pathogen, and no more than two blood cultures (taken on different dates) positive for the baseline blood pathogen. Clinical success at end of treatment (EOT) in these studies was typically defined as resolution of signs and symptoms of the presenting illness (e.g., a decrease in lesion area, no fever, and local signs of fluctuance/warmth absent [DUR001-301 and DUR001-302] or improved and no worse than mild [DUR001-303]), tenderness/swelling/induration no worse than mild, purulent drainage improved and no worse than mild, and no requirement for new systemic or concurrent antibiotics. Clinical success for the treatment of catheter-related bloodstream infection or cSSSIs was defined as resolution of all signs and symptoms of the presenting illness, with no requirement for new systemic antibiotics [VER001-4 and VER001-9] and no fever [VER001-4] (Table 1). 


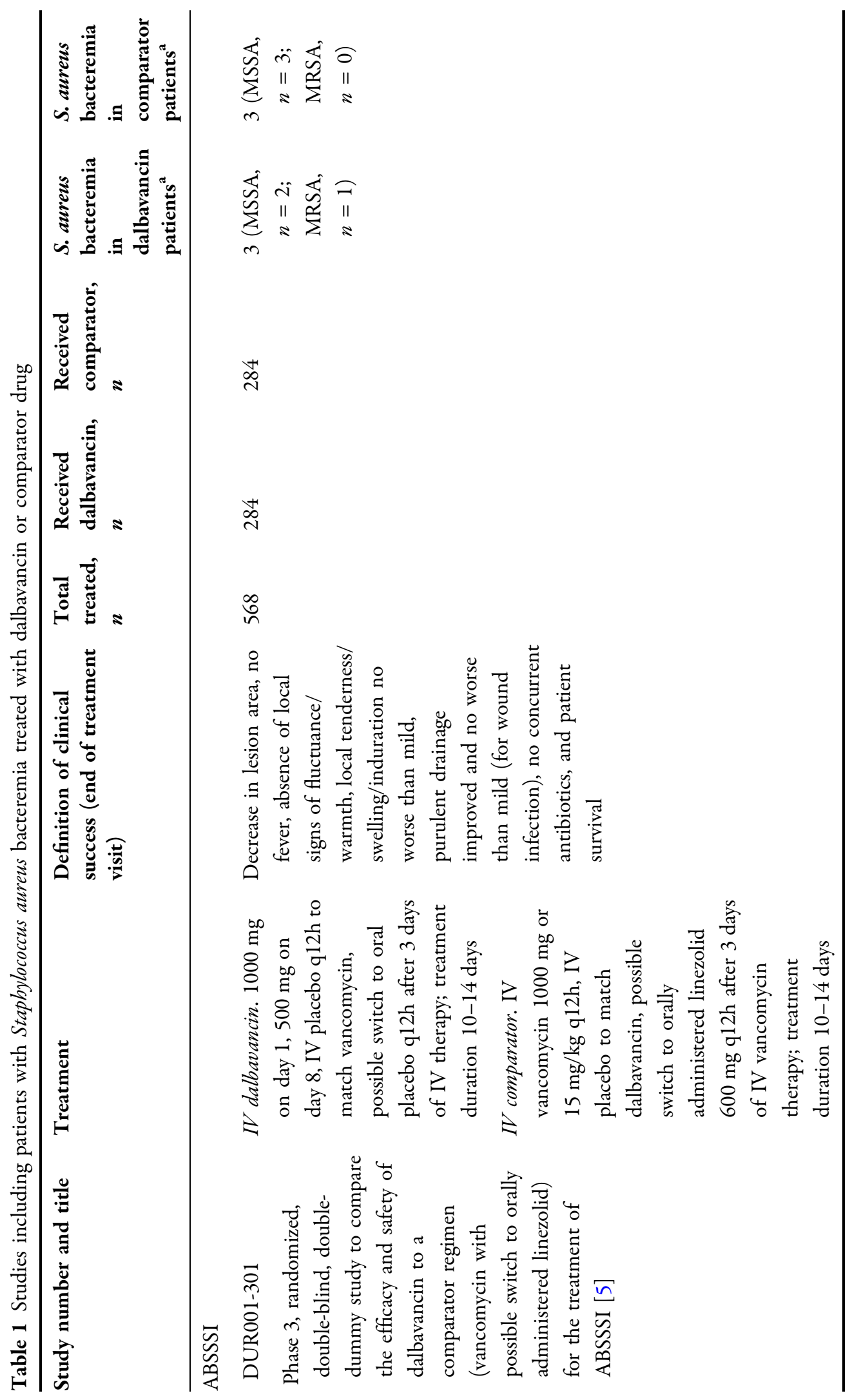




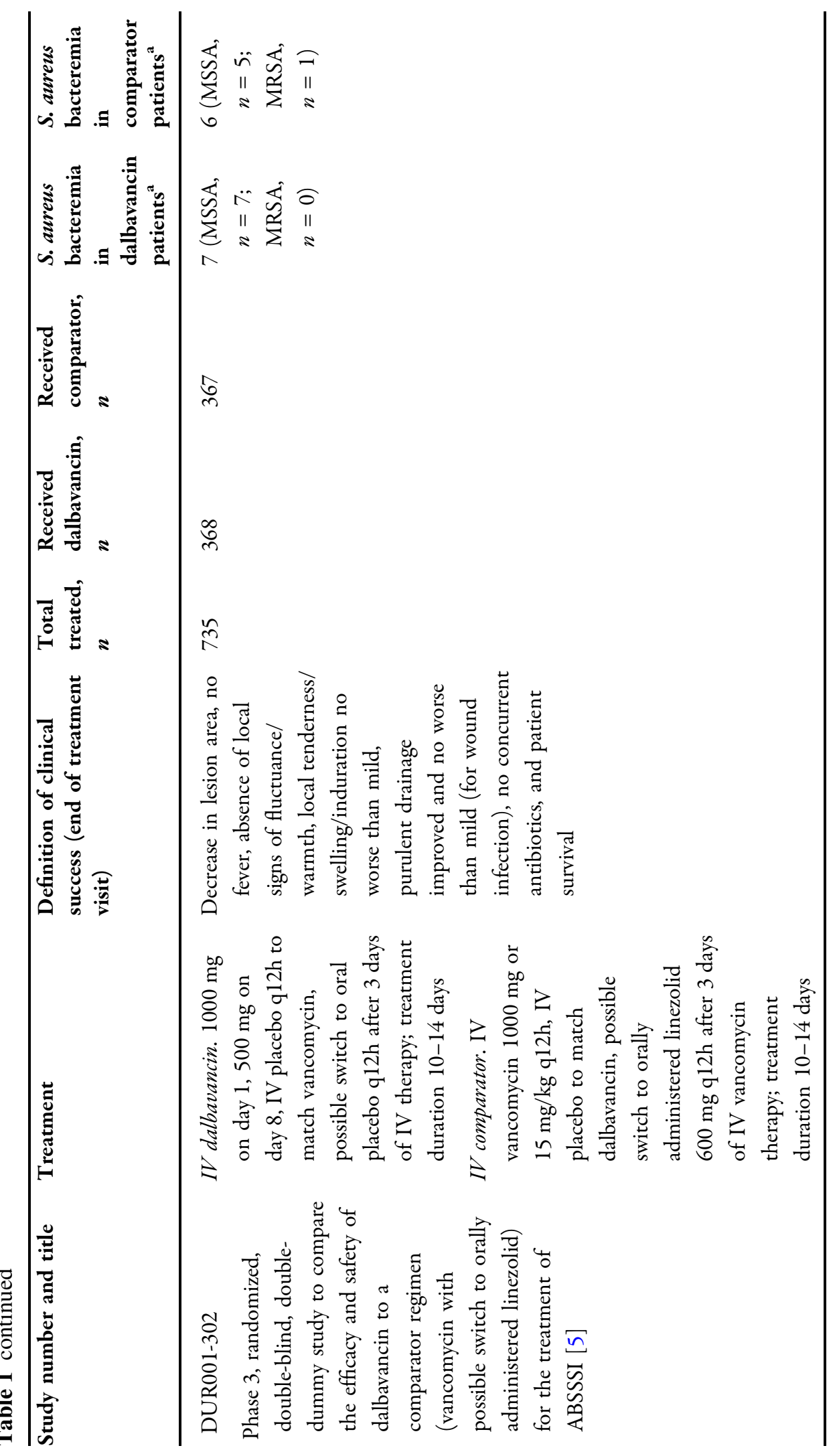




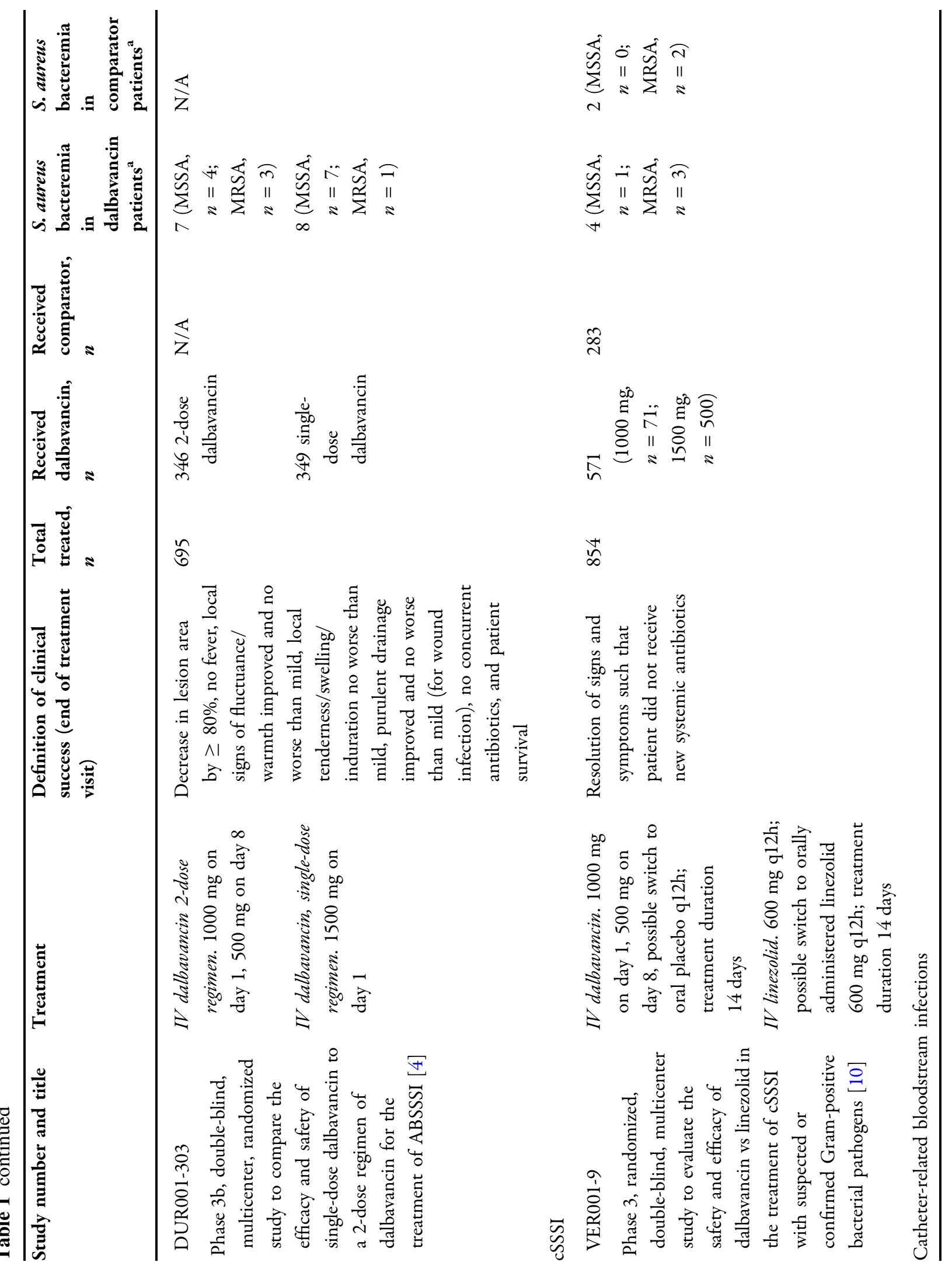




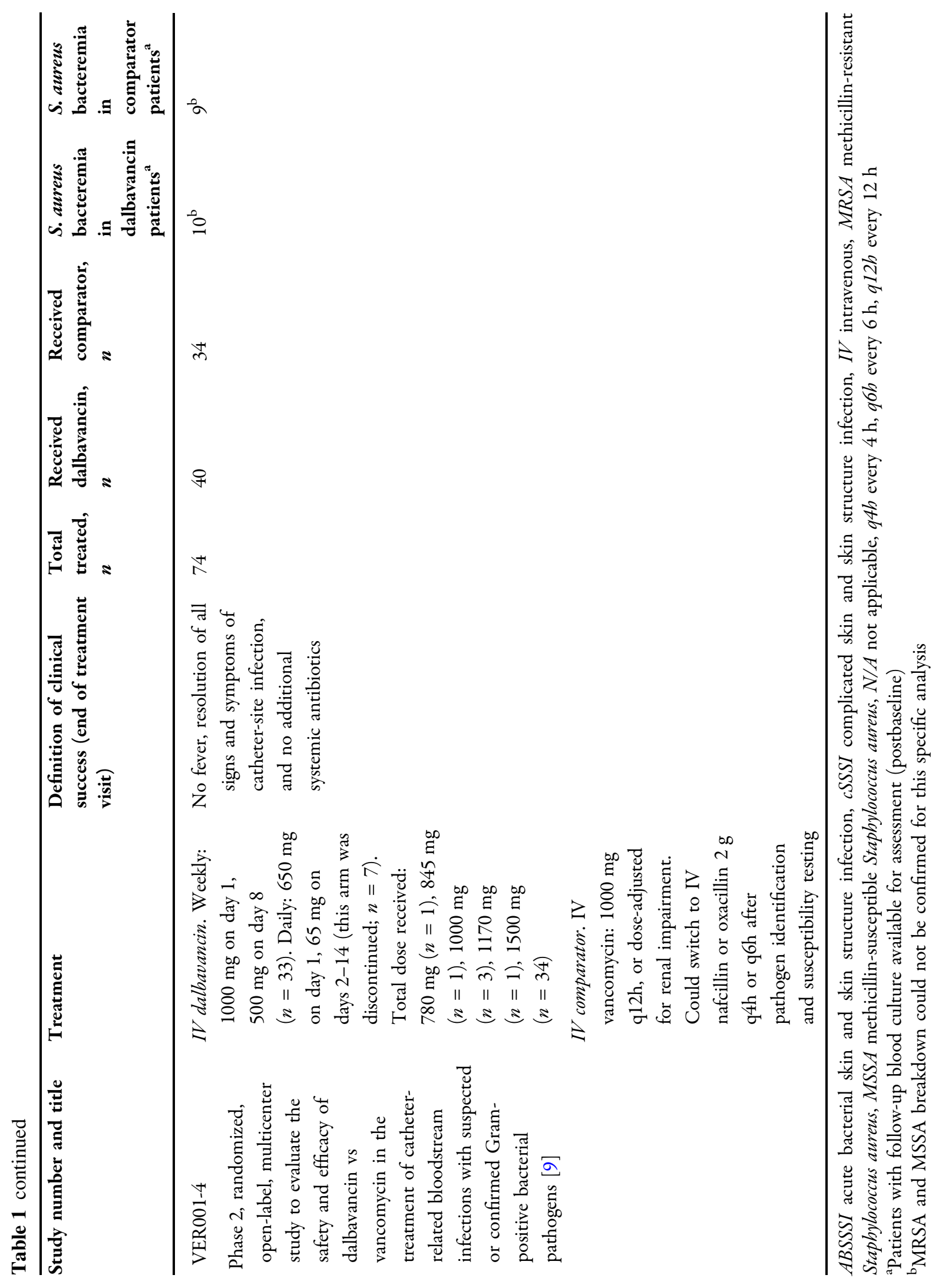


Table 2 Documented clearance and clinical outcomes in patients with Staphylococcus aureus bacteremia receiving dalbavancin or comparator drug

\begin{tabular}{|c|c|c|c|c|c|c|}
\hline \multirow{2}{*}{$\begin{array}{l}\text { Infection, } \\
n / N\end{array}$} & \multicolumn{3}{|l|}{ Dalbavancin } & \multicolumn{3}{|l|}{ Comparator } \\
\hline & $\begin{array}{l}\text { Clearance of } \\
\text { bacteremia }^{\text {a }}\end{array}$ & $\begin{array}{l}\geq 20 \% \text { Reduction in } \\
\text { lesion size at } \\
48-72 \mathrm{~h}\end{array}$ & $\begin{array}{l}\text { Clinical } \\
\text { success }^{b} \text { at } \\
\text { EOT }\end{array}$ & $\begin{array}{l}\text { Clearance } \\
\text { of } \\
\text { bacteremia }^{a}\end{array}$ & $\begin{array}{l}\geq 20 \% \text { Reduction in } \\
\text { lesion size at } \\
48-72 \mathrm{~h}\end{array}$ & $\begin{array}{l}\text { Clinical } \\
\text { success }{ }^{b} \text { at } \\
\text { EOT }\end{array}$ \\
\hline \multicolumn{7}{|l|}{ ABSSSI } \\
\hline $\begin{array}{l}\text { DUR001- } \\
301\end{array}$ & $3 / 3$ & $3 / 4$ & $2 / 3$ & $2 / 3$ & $1 / 3$ & $3 / 3$ \\
\hline $\begin{array}{l}\text { DUR001- } \\
302\end{array}$ & $7 / 7$ & $6 / 7$ & $5 / 6$ & $6 / 6$ & $5 / 6$ & $5 / 6$ \\
\hline $\begin{array}{l}\text { DUR001- } \\
303\end{array}$ & $15 / 15$ & $14 / 15$ & $14 / 14$ & $\mathrm{~N} / \mathrm{A}$ & N/A & $\mathrm{N} / \mathrm{A}$ \\
\hline \multicolumn{7}{|l|}{ cSSSI } \\
\hline VER001-9 & $4 / 4$ & - & $3 / 4$ & $2 / 2$ & - & $2 / 2$ \\
\hline \multicolumn{7}{|c|}{ Catheter-related bloodstream infections } \\
\hline VER001-4 & $10 / 10$ & - & $9 / 9$ & $9 / 9$ & - & $8 / 12$ \\
\hline $\begin{array}{r}\text { Total, } n / \\
N(\%)^{\mathrm{c}}\end{array}$ & $39 / 39(100)$ & $23 / 26(88)$ & $33 / 36(92)$ & $19 / 20(95)$ & $6 / 9(67)$ & $18 / 23(78)$ \\
\hline
\end{tabular}

ABSSSI acute bacterial skin and skin structure infection, $C S S S I$ complicated skin and skin structure infection, EOT end of treatment, $N / A$ not applicable (no comparator drug)

${ }^{\text {a }}$ Patients with a follow-up blood culture available for assessment (postbaseline)

${ }^{\mathrm{b}}$ Clinically evaluable population (those with missing data excluded from the analysis) of patients with a positive blood culture at baseline

${ }^{\mathrm{c}}$ Difference between treatment groups is 5 (95\% CI - 4, 24). Difference refers to difference in bacteremia clearance rates (dalbavancin treatment group minus comparator treatment group). CIs are calculated using the Miettinen-Nurminen method without adjustments

\section{RESULTS}

Fifty-nine patients with $S$. aureus bacteremia at baseline had follow-up blood cultures available for assessment. Thirt-nine patients were treated with dalbavancin; eight of these patients received dalbavancin $1500 \mathrm{mg}$ as a single dose. Twenty patients received a comparator drug, vancomycin or linezolid (Table 1 ).

The clinical studies pooled for analysis included patients with $S$.aureus bacteremia, including MRSA. S.aureus cleared in all 39 patients $(100 \%)$ who were treated with dalbavancin and who had follow-up blood cultures available for assessment. By comparison,
S. aureus bacteremia cleared in $19 / 20$ patients (95\%) treated with comparator (Table 2) and in all eight patients receiving single-dose dalbavancin.

None of the clinical failures in the dalbavancin group resulted from persistent underlying bacteremia. The reasons for clinical failure in patients receiving dalbavancin included local signs of tenderness to palpation and swelling/ induration that were worse than mild, local signs of fluctuance and localized heat/warmth that had not resolved, receipt of a concomitant antibiotic, or unplanned surgical intervention for ABSSSI more than $72 \mathrm{~h}$ after study drug administration. 
In the ABSSSI studies, a greater proportion of patients in the dalbavancin group had at least a $20 \%$ reduction in lesion size $48-72 \mathrm{~h}$ after the start of treatment in $23 / 26$ patients $(88 \%)$ compared with $6 / 9$ patients $(67 \%)$ in the comparator group. Across all five studies, patients in the dalbavancin group had higher rates of clinical success at EOT (33/36 patients [92\%]) than those in the comparator group $(18 / 23$ patients [78\%]).

\section{DISCUSSION}

In this analysis of phase 2 and 3 clinical trial data, all 39 patients with uncomplicated $S$. aureus bacteremia at baseline who were treated with dalbavancin, either as a single dose or a two-dose regimen, and had follow-up blood cultures available for assessment had clearance of their bloodstream infection. Clinical outcomes in patients receiving either a single-dose or two-dose dalbavancin regimen were similar to those of patients receiving daily treatment with comparator drugs for 10-14 days.

The clinical success rate of dalbavancin in this pooled analysis was higher than vancomycin, linezolid, or daptomycin, as reported in other clinical trials of S. aureus bacteremia, although the patient populations and definitions of success differed between the studies. In a pooled meta-analysis of patients with $S$. aureus bacteremia, the clinical success rates of vancomycin and linezolid were 52\% and 55\%, respectively, with microbiological success in $73 \%$ and $69 \%$ of patients [11]. In addition, in an open-label randomized trial, the clinical success rate of daptomycin in the treatment of uncomplicated S.aureus bacteremia was $56 \%$ [12]. In an open-label study that investigated catheter-related bloodstream infections in patients with complicated skin and skin structure infections, the clinical success was $82 \%$ and $83 \%$ in the linezolid and vancomycin groups, respectively, with microbiologic success occurring in $67 \%$ of patients in each group [13].

Recent anecdotal reports of the efficacy of dalbavancin in bloodstream infections have been published [14-26]. A retrospective cohort study conducted in the University Hospital of
Vienna between January 2015 and December 2016 showed that microbiological and clinical success was achieved in 93\% (25/27) of patients who received dalbavancin either as primary or sequential treatment for infective endocarditis. In these patients, the pathogens were predominantly S. aureus $(n=9)$ and E. faecalis $(n=4)$ [18]. Another retrospective study described the efficacy and safety of dalbavancin in 69 adult patients (catheter-related bacteremia, $n=8$; endocarditis, $n=7$ ) treated between 2016 and 2017 across 29 institutions in Spain. In patients with catheter-related bacteremia, the pathogens were MRSA $(n=3)$, coagulase-negative staphylococci (CoNS) $(n=3)$, methicillin-susceptible S. aureus (MSSA; $n=2$ ), and Enterococcus spp. $(n=1)$; in patients with endocarditis, the pathogens were CoNS $(n=2)$, Enterococcus spp. $(n=2)$, MRSA $(n=1)$, and Streptococcus spp. $(n=1)$. Clinical success was achieved for $75 \%$ of patients with catheter-related bacteremia and $86 \%$ of patients with endocarditis [20]. Additional retrospective studies have been published describing successful treatment of Gram-positive bacteremia and/or endocarditis with dalbavancin [14, 15, 17, 22, 24-26].

Four case reports described successful treatment with dalbavancin $[16,19,21,23]$ : the first described successful treatment of catheter-associated bloodstream infection due to $E$. faecalis in an intravenous drug user [19], the second showed successful treatment of a 54-year-old man with MSSA bacteremia secondary to septic phlebitis [21], the third showed successful treatment of a 27-year-old woman with MRSA tricuspid-valve endocarditis with septic pulmonary emboli [16], and the fourth showed successful treatment of an 88-year-old woman with MRSA prosthetic vascular graft infection [23]. A phase $2 \mathrm{~b}$ multicenter randomized clinical trial (NCT04775953) that is currently enrolling patients will compare the safety and efficacy of dalbavancin with standard-of-care antibiotics for the completion of therapy of complicated $S$. aureus bacteremia or right-sided native valve infective endocarditis in patients who have cleared their bacteremia [27].

Limitations of this study include the fact that clinical success was defined differently across the studies, primarily owing to evolving 
definitions in regulatory guidance for skin infection. The more recent studies used the newer definition of clinical success or the improvement and resolution of clinical signs and symptoms, such as pain or reduction in lesion size. These studies also used an updated definition of ABSSSI [28]. Another limitation is the small sample size of patients with $S$. aureus bacteremia at baseline, an expected finding given that four of the five trials enrolled patients with skin infection and that, for the 15 patients with $S$. aureus bacteremia in DUR001303 randomized to either single- or two-dose dalbavancin, there was no corresponding control group receiving comparator antibiotics.

\section{CONCLUSIONS}

Dalbavancin is indicated for treatment of adults with ABSSSI caused by designated susceptible strains of Gram-positive microorganisms and offers an alternative to treat $S$. aureus bacteremia in these patients. Dalbavancin can be given either as a single dose of $1500 \mathrm{mg}$ or as a 1000 -mg dose followed 1 week later by $500 \mathrm{mg}$. The current subgroup analysis helps lay the groundwork for a randomized controlled trial further evaluating the efficacy of dalbavancin in the treatment of $S$. aureus bacteremia.

\section{ACKNOWLEDGEMENTS}

Funding. This work was supported by Allergan (before its acquisition by AbbVie). The study sponsor is also funding the journal's Rapid Service fees.

Medical Writing, Editorial, and Other Assistance. The authors thank Kalyan Tadikonda for his contribution to the development of this manuscript. Editorial support was provided by Lee B. Hohaia, PharmD, Jennifer L. Venzie, PhD, and John E. Fincke, PhD, at ICON plc (North Wales, PA) and was funded by Allergan plc (Dublin, Ireland; before its acquisition by AbbVie).
Authorship. All named authors meet the International Committee of Medical Journal Editors (ICMJE) criteria for authorship for this article, take responsibility for the integrity of the work as a whole, and have given their approval for this version to be published.

Author Contributions. All authors contributed to the study conception and design. Material preparation, data collection and analysis were performed by KA, MWD, SP and UR. The first draft of the manuscript was written by UR, and all authors commented on subsequent versions of the manuscript. All authors read and approved the final manuscript.

Prior Presentations. European Congress of Clinical Microbiology and Infectious Diseases (ECCMID); Vienna, Austria; 22-25 April 2017. Making a Difference in Infectious Diseases (MAD-ID); Orlando, FL, USA; 9-12 May 2018.

Disclosures. Pedro L. Gonzalez was an employee of AbbVie at the time of study conduct and analysis and is a current employee of Becton-Dickinson (Franklin Lakes, NJ). Urania Rappo was an employee of Allergan (prior to its acquisition by AbbVie) at the time of study conduct and analysis and is a current employee of BiomX Inc. (Branford, CT). Karthik Akinapelli and Michael W. Dunne were employees of Allergan (prior to its acquisition by AbbVie) at the time of study conduct and are current employees of Iterum Therapeutics (Old Saybrook, CT). Jennifer S. McGregor was an employee of AbbVie at the time of study conduct and may hold AbbVie stock. Sailaja Puttagunta was an employee of Allergan (prior to its acquisition by AbbVie) at the time of study conduct and held stock in the company and is a current employee of BiomX Inc. (Branford, CT). All authors met the ICMJE authorship criteria. Neither honoraria nor payments were made for authorship.

Compliance with Ethics Guidelines. This article is based on previously conducted studies, which were performed in accordance with the Declaration of Helsinki, the study protocol, the International Conference on Harmonisation 
tripartite guideline for Good Clinical Practice (ICH E6[R1]), and the institutional review board or ethics committee at each study site.

Data Availability. The datasets generated and/or analyzed during the current study are available from the corresponding author on reasonable request.

Open Access. This article is licensed under a Creative Commons Attribution-NonCommercial 4.0 International License, which permits any non-commercial use, sharing, adaptation, distribution and reproduction in any medium or format, as long as you give appropriate credit to the original author(s) and the source, provide a link to the Creative Commons licence, and indicate if changes were made. The images or other third party material in this article are included in the article's Creative Commons licence, unless indicated otherwise in a credit line to the material. If material is not included in the article's Creative Commons licence and your intended use is not permitted by statutory regulation or exceeds the permitted use, you will need to obtain permission directly from the copyright holder. To view a copy of this licence, visit http:// creativecommons.org/licenses/by-nc/4.0/.

\section{REFERENCES}

1. Economou NJ, Nahoum V, Weeks SD, et al. A carrier protein strategy yields the structure of dalbavancin. J Am Chem Soc. 2012;134:4637-45.

2. Dalvance ${ }^{\circledR}$ (dalbavancin). Full Prescribing Information. Parsippany: Durata Therapeutics US Ltd.; 2021.

3. Xydalba (dalbavancin). Summary of product characteristics. Amsterdam: Durata Therapeutics International BV; 2017.

4. Dunne MW, Puttagunta S, Giordano P, et al. A randomized clinical trial of single-dose versus weekly dalbavancin for treatment of acute bacterial skin and skin structure infection. Clin Infect Dis. 2016;62:545-51.

5. Boucher HW, Wilcox M, Talbot GH, et al. Onceweekly dalbavancin versus daily conventional therapy for skin infection. N Engl J Med. 2014;370: 2169-79.

6. Mendes RE, Castanheira M, Farrell DJ, et al. Update on dalbavancin activity tested against Gram-positive clinical isolates responsible for documented skin and skin-structure infections in US and European hospitals (2011-13). J Antimicrob Chemother. 2016;71:276-8.

7. Dorr MB, Jabes D, Cavaleri M, et al. Human pharmacokinetics and rationale for once-weekly dosing of dalbavancin, a semi-synthetic glycopeptide. J Antimicrob Chemother. 2005;55:ii25-30.

8. Dunne MW, Talbot GH, Boucher HW, Wilcox M, Puttagunta S. Safety of dalbavancin in the treatment of skin and skin structure infections: a pooled analysis of randomized, comparative studies. Drug Saf. 2016;39:147-57.

9. Raad I, Darouiche R, Vazquez J, et al. Efficacy and safety of weekly dalbavancin therapy for catheterrelated bloodstream infection caused by Grampositive pathogens. Clin Infect Dis. 2005;40: 374-80.

10. Jauregui LE, Babazadeh S, Seltzer E, et al. Randomized, double-blind comparison of once-weekly dalbavancin versus twice-daily linezolid therapy for the treatment of complicated skin and skin structure infections. Clin Infect Dis. 2005;41:1407-15.

11. Shorr AF, Kunkel MJ, Kollef M. Linezolid versus vancomycin for Staphylococcus aureus bacteraemia: pooled analysis of randomized studies. J Antimicrob Chemother. 2005;56:923-9.

12. Fowler VG, Boucher HW, Corey GR, et al. Daptomycin versus standard therapy for bacteremia and endocarditis caused by Staphylococcus aureus. N Engl J Med. 2006;355:653-65.

13. Wilcox MH, Tack KJ, Bouza E, et al. Complicated skin and skin-structure infections and catheter-related bloodstream infections: noninferiority of linezolid in a phase 3 study. Clin Infect Dis. 2009;48:203-12.

14. Streifel AC, Sikka MK, Bowen CD, Lewis JS 2nd. Dalbavancin use in an academic medical centre and associated cost savings. Int J Antimicrob Agents. 2019;54:652-4.

15. Hidalgo-Tenorio C, Vinuesa D, Plata A, et al. DALBACEN cohort: dalbavancin as consolidation therapy in patients with endocarditis and/or bloodstream infection produced by Gram-positive cocci. Ann Clin Microbiol Antimicrob. 2019;18:30.

16. Hakim A, Braun H, Thornton D, Strymish J. Successful treatment of methicillin-sensitive 
Staphylococcus aureus tricuspid-valve endocarditis with dalbavancin as an outpatient in a person who injects drugs: a case report. Int J Infect Dis. 2020;91: 202-5.

17. Dinh A, Duran C, Pavese P, et al. French national cohort of first use of dalbavancin: a high proportion of off-label use. Int J Antimicrob Agents. 2019;54: 668-72.

18. Tobudic S, Forstner C, Burgmann H, et al. Dalbavancin as primary and sequential treatment for Gram-positive infective endocarditis: 2-year experience at the General Hospital of Vienna. Clin Infect Dis. 2018;67:795-8.

19. Jones BM, Keedy C, Wynn M. Successful treatment of Enterococcus faecalis bacteremia with dalbavancin as an outpatient in an intravenous drug user. Int J Infect Dis. 2018;76:4-5.

20. Bouza E, Valerio M, Soriano A, et al. Dalbavancin in the treatment of different Gram-positive infections: a real-life experience. Int $\mathrm{J}$ Antimicrob Agents. 2018;51:571-7.

21. Cho JC, Estrada SJ, Beltran AJ, Revuelta MP. Treatment of methicillin-sensitive Staphylococcus aureus bacteremia secondary to septic phlebitis using dalbavancin. J Clin Pharm Ther. 2015;40:604-6.

22. Bork JT, Heil EL, Berry S, et al. Dalbavancin use in vulnerable patients receiving outpatient parenteral antibiotic therapy for invasive Gram-positive infections. Infect Dis Ther. 2019;8:171-84.

23. Ciccullo A, Giuliano G, Segala FV, et al. Dalbavancin as a second-line treatment in methicillinresistant Staphylococcus aureus prosthetic vascular graft infection. Infection. 2020;48:309-10.
24. Morrisette T, Miller MA, Montague BT, et al. Longacting lipoglycopeptides: "lineless antibiotics" for serious infections in persons who use drugs. Open Forum Infect Dis. 2019;6:ofz274.

25. Bryson-Cahn C, Beieler AM, Chan JD, Harrington $\mathrm{RD}$, Dhanireddy S. Dalbavancin as secondary therapy for serious Staphylococcus aureus infections in a vulnerable patient population. Open Forum Infect Dis. 2019. https://doi.org/10.1093/ofid/ofz028.

26. Wunsch S, Krause R, Valentin T, et al. Multicenter clinical experience of real life dalbavancin use in Gram-positive infections. Int J Infect Dis. 2019;81: 210-4.

27. Antibacterial Resistance Leadership Group. DOTS: Dalbavancin as an option for treatment of $S$. aureus bacteremia. National Institute of Allergy and Infectious Diseases. 2021. https://arlg.org/studiesin-progress. Accessed 15 Feb 2021.

28. US Department of Health and Human Services, US Food and Drug Administration, Center for Drug Evaluation and Research (CDER). Guidance for industry. Acute bacterial skin and skin structure infections: developing drugs for treatment. 2021. https://www.fda.gov/files/drugs/published/AcuteBacterial-Skin-and-Skin-Structure-InfectionsDeveloping-Drugs-for-Treatment.pdf. Accessed 15 Feb 2021.

\section{Publisher's Note}

Springer Nature remains neutral with regard to jurisdictional claims in published maps and institutional affiliations 